\title{
Thermodynamic limit and boundary energy of the spin-1 Heisenberg chain with non-diagonal boundary fields
}

\author{
Zhihan Zheng ${ }^{1,2}$, Pei Sun ${ }^{1,2}$, Xiaotian $\mathrm{Xu}^{1,2 \star}$, Tao Yang ${ }^{1,2,3,4}$, \\ Junpeng Cao ${ }^{4,5,6,7}$ and Wen-Li Yang ${ }^{1,2,3,4}$
}

1 Institute of Modern Physics, Northwest University, Xi'an 710127, China

2 Shaanxi Key Laboratory for Theoretical Physics Frontiers, Xi'an 710127, China

3 School of Physics, Northwest University, Xi'an 710127, China

4 Peng Huanwu Center for Fundamental Theory, Xi'an 710127, China

5 Beijing National Laboratory for Condensed Matter Physics, Institute of Physics,

Chinese Academy of Sciences, Beijing 100190, China

6 Songshan Lake Materials Laboratory, Dongguan, Guangdong 523808, China

7 School of Physical Sciences, University of Chinese Academy of Sciences, Beijing 100049, China

$\star x t x u @ n w u . e d u . c n$

\begin{abstract}
The thermodynamic limit and boundary energy of the isotropic spin-1 Heisenberg chain with non-diagonal boundary fields are studied. The finite size scaling properties of the inhomogeneous term in the $T-Q$ relation at the ground state are calculated by the density matrix renormalization group. Based on our findings, the boundary energy of the system in the thermodynamic limit can be obtained from Bethe ansatz equations of a related model with parallel boundary fields. These results can be generalized to the $S U(2)$ symmetric high spin Heisenberg model directly.
\end{abstract}

(c) (1) Copyright Z. Zheng et al.

This work is licensed under the Creative Commons

Attribution 4.0 International License.

Published by the SciPost Foundation.
Received 02-06-2021

Accepted 13-01-2022

Published 22-02-2022

doi:10.21468/SciPostPhys.12.2.071

\section{Contents}

1 Introduction $\quad 2$

2 Non-diagonal boundary Spin-1 Heisenberg model 3

$\begin{array}{llr}3 & \text { Finite size scaling behavior } & 6\end{array}$

4 Boundary energy $\quad 11$

5 Conclusions $\quad 15$

$\begin{array}{ll}\text { References } & 16\end{array}$ 


\section{Introduction}

The study of quantum integrable models is an interesting subject in the fields of cold atoms, quantum field theory, condensed matter physics and statistic mechanics [1-5]. The spin-1/2 Heisenberg model can effectively quantify the spin-exchanging interaction and plays an important role in the quantum magnetism and many-body theory. By using the Bethe ansatz method, the one-dimensional (1D) spin-1/2 Heisenberg model can be solved exactly [6]. The typical spin-exchanging couplings in the 1D spin-1 system are characterized by the bilinear biquadratic model, where the Hamiltonian reads

$$
H=\sum_{k=1}^{N}\left[J_{1} \vec{S}_{k} \cdot \vec{S}_{k+1}+J_{2}\left(\vec{S}_{k} \cdot \vec{S}_{k+1}\right)^{2}\right] .
$$

Here $\vec{S}_{k}\left(S_{k}^{x}, S_{k}^{y}, S_{k}^{z}\right)$ is the spin-1 operator at site $k, N$ is the number of sites, and the periodic boundary condition gives $\vec{S}_{N+1}=\vec{S}_{1}$. If $J_{2} / J_{1}=1$, the system (1) has the $S U(3)$ symmetry and is integrable. If $J_{2} / J_{1}=-1$, the $S U(2)$ symmetry exists, and the system is known as the Zamalodchikov-Fateev (ZF) model [7]. The Bethe ansatz solution and thermodynamic properties of the ZF model are studied by Takhtajan [8] and Babujian $[9,10]$. If $J_{2}=0$, the system is no longer integrable. Starting from the nonlinear sigma model, Haldane conjectures that the excitation of the system has a gap $[11,12]$. If $J_{2} / J_{1}=1 / 3$, the Hamiltonian (1) degenerates into a projector operator that is in fact the projection onto the sum of the spin-0 and spin- 1 subspaces (up to a constant) and the ground state is the famous valence bond solid state $[13,14]$. If $J_{1}=0$, by using the Temperley-Lieb algebra, the system can be mapped into the XXZ spin chain and is also integrable [15-17].

Besides the periodic boundary condition, the integrable open one is also an interesting subject, which means that the system has magnetic impurity or the boundary magnetic fields $[18,19]$. In the past few decades, the exact results of high spin models with periodic [7-10, 20-25] and parallel boundary fields [26-29] have been extensively studied. It is emphasized that the integrable boundary reflection matrix can have non-diagonal elements, which means that the boundary fields are unparallel. Then the $U(1)$ symmetry is broken and it is very hard to study the exact solution of the system. It is known that the integrable systems without $U(1)$ symmetry have many applications in the open string theory and the stochastic process of nonequilibrium statistics. Therefore, many interesting works of high spin models with nondiagonal boundary reflections have been done [30-35].

Many attentions have been paid for quantum integrable models without $U(1)$ symmetry during past decades [36-49]. Recently, a systematic method, i.e., the off-diagonal Bethe ansatz (ODBA) is proposed to solve the models with or without $U(1)$ symmetry [50]. Eigenvalues and eigenstates of several typical integrable models are obtained, where eigenvalues are given in terms of some homogeneous/inhomogeneous $T-Q$ relation [50-53]. The next task is to derive the physical quantities in the thermodynamic limit, which is very complicated because the related Bethe ansatz equations (BAEs) are inhomogeneous and the traditional thermodynamic Bethe ansatz can not be employed. In order to overcome this difficulty, an effective method is to study the finite size scaling effects of the inhomogeneous term in the $T-Q$ relation. With the help of this idea, the thermodynamic limit, surface energy and elementary excitations of spin-1/2 XXZ spin chain with arbitrary boundary fields are studied [54]. The boundary energy of the $S U(3)$ symmetric spin-1 chain with generic integrable open boundaries is also obtained [55]. However, the corresponding thermodynamic properties of the $S U(2)$ symmetric spin-1 Heisenberg model are still missing.

In this paper, we study the thermodynamic limit and boundary energy of the spin- 1 isotropic Heisenberg spin chain with non-diagonal boundary reflections. The finite size scaling analysis of the contribution of the inhomogeneous term in the $T-Q$ relation (namely, the third 
term in (18) below) to the ground state energy is studied as follows. We first introduce a very function $\Lambda_{\text {hom }}(u)$ which is given in terms of a reduced $T-Q$ relation ${ }^{1}$ (see (27) and (28) below) [51-53] and the associated BAEs are homogeneous ones (see (29) below). For any finite $N, \Lambda_{\text {hom }}(u)$ is actually not an eigenvalue of the transfer matrix with generic off-diagonal boundary $K$-matrices. Since that the function is given by a homogeneous $T-Q$ relation, we can apply the conventional thermodynamic Bethe ansatz [2] to investigate its thermodynamic limit. Then, comparing with the result of its thermodynamic limit and that of the density matrix renormalization group (DMRG) numerical [56-58] studies, we conclude that $\Lambda_{\text {hom }}(u)$, in the limit $N \rightarrow \infty$, really gives the correct boundary energy. Moreover, we find that most Bethe roots of the reduced BAEs at the ground state in the thermodynamic limit form 2-strings, associated with certain boundary strings and the rearrangement of the Fermi sea. The different structures of Bethe roots in different regimes of model parameters are given explicitly. Based on them, we obtain the boundary energy induced by the boundary magnetic fields. We also check the analytic results by the numerical extrapolation, and find that the analytical results and the numerical ones coincide with each other very well. The results given in this paper can be generalized to the $S U(2)$ symmetric spin-s Heisenberg model directly.

This paper is organized as follows. Section 2 serves as an introduction to the notations for the spin-1 Heisenberg model with non-diagonal boundary fields. The ODBA exact solution is also briefly reviewed. In Section 3, we focus on the contribution of the inhomogeneous term in the $T-Q$ relation to the ground state energy. In Section 4, by using the patterns of Bethe roots of the reduced BAEs, we study the boundary energy of the model in the thermodynamic limit. We summarize the results and give some discussions in Section 5.

\section{Non-diagonal boundary Spin-1 Heisenberg model}

The spin-1 Heisenberg model with non-diagonal boundary fields is related to the 19-vertex $R$-matrix

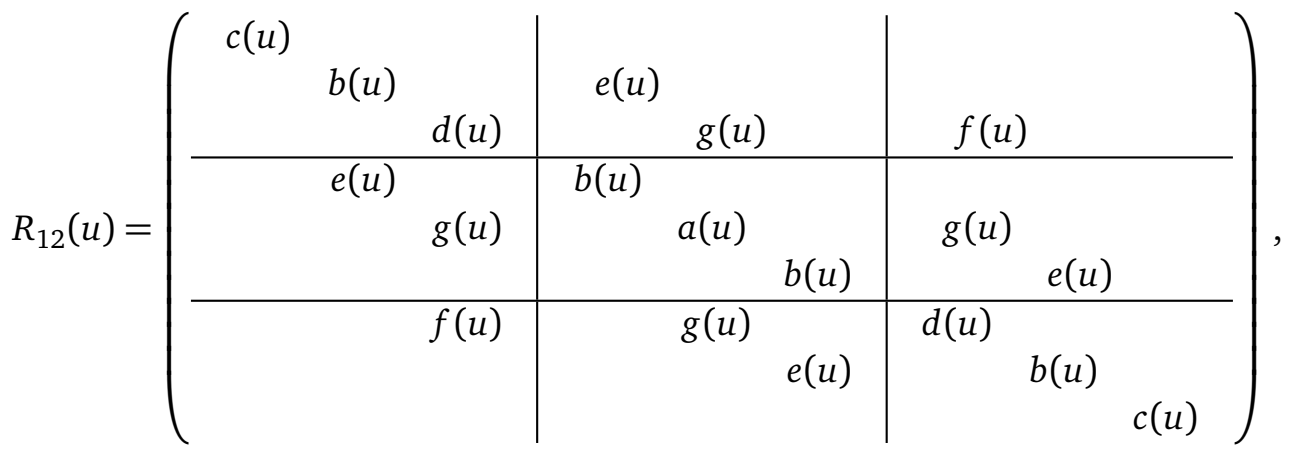

where the non-vanishing elements are

$$
\begin{gathered}
a(u)=u(u+\eta)+2 \eta^{2}, b(u)=u(u+\eta), c(u)=(u+\eta)(u+2 \eta), \\
d(u)=u(u-\eta), e(u)=2 \eta(u+\eta), f(u)=2 \eta^{2}, g(u)=2 u \eta
\end{gathered}
$$

$u$ is the spectral parameter, and $\eta$ is the crossing parameter. Here we are dealing with the isotropic model, and $\eta$ can be scaled out. Throughout this paper, we adopt the standard notations. For any matrix $A \in \operatorname{End}(\mathbb{V}), A_{j}$ is an embedding operator in the tensor space $\mathbb{V} \otimes \mathbb{V} \otimes \ldots$, which acts as $A$ on the $j$-th space and as identity on the other factor spaces. For any matrix $B \in \operatorname{End}(\mathbb{V} \otimes \mathbb{V}), B_{i, j}$ is an embedding operator in the tensor space, which acts as an identity on

\footnotetext{
${ }^{1}$ The function $\Lambda_{\text {hom }}(u)$ can be simulated by eigenvalue of the transfer matrix with parallel boundary fields of the strengthes: $p \rightarrow p / \sqrt{1+\alpha_{-}^{2}} ; q \rightarrow q / \sqrt{1+\alpha_{+}^{2}}$.
} 
the factor spaces except for the $i$-th and $j$-th ones. The $R$-matrix $R_{12}(u)$ satisfies the quantum Yang-Baxter equation (QYBE) $[59,60]$

$$
R_{12}(u-v) R_{13}(u) R_{23}(v)=R_{23}(v) R_{13}(u) R_{12}(u-v) .
$$

Besides, the $R$-matrix (2) also enjoys the properties

$$
\begin{aligned}
& \text { Initial condition : } R_{12}(0)=2 \eta^{2} P_{12}, \\
& \text { Fusion condition }: R_{12}(-\eta)=6 \eta^{2} \mathbf{P}_{12}^{(0)}
\end{aligned}
$$

where $P_{12}$ is the permutation operator and $\mathbf{P}_{12}^{(0)}$ is the projector in the total spin-0 channel. The most general off-diagonal boundary reflection on one side of the chain is quantified by the reflection matrix obtained in $[61,62]$

$$
K^{-}(u)=(2 u+\eta)\left(\begin{array}{lll}
x_{1}(u) & y_{4}^{\prime}(u) & y_{6}^{\prime}(u) \\
y_{4}(u) & x_{2}(u) & y_{5}^{\prime}(u) \\
y_{6}(u) & y_{5}(u) & x_{3}(u)
\end{array}\right),
$$

where the matrix elements are

$$
\begin{aligned}
& x_{1}(u)=\left(p_{-}+u+\frac{\eta}{2}\right)\left(p_{-}+u-\frac{\eta}{2}\right)+\frac{\alpha_{-}^{2}}{2} \eta\left(u-\frac{\eta}{2}\right), \\
& x_{2}(u)=\left(p_{-}+u-\frac{\eta}{2}\right)\left(p_{-}-u+\frac{\eta}{2}\right)+\alpha_{-}^{2}\left(u+\frac{\eta}{2}\right)\left(u-\frac{\eta}{2}\right), \\
& x_{3}(u)=\left(p_{-}-u-\frac{\eta}{2}\right)\left(p_{-}-u+\frac{\eta}{2}\right)+\frac{\alpha_{-}^{2}}{2} \eta\left(u-\frac{\eta}{2}\right), \\
& y_{4}(u)=\sqrt{2} \alpha_{-} e^{-i \phi_{-}} u\left(p_{-}+u-\frac{\eta}{2}\right), \quad y_{4}^{\prime}(u)=\sqrt{2} \alpha_{-} e^{i \phi_{-}} u\left(p_{-}+u-\frac{\eta}{2}\right), \\
& y_{5}(u)=\sqrt{2} \alpha_{-} e^{-i \phi_{-}} u\left(p_{-}-u+\frac{\eta}{2}\right), \quad y_{5}^{\prime}(u)=\sqrt{2} \alpha_{-} e^{i \phi_{-}} u\left(p_{-}-u+\frac{\eta}{2}\right), \\
& y_{6}(u)=\alpha_{-}^{2} e^{-2 i \phi_{-}} u\left(u-\frac{\eta}{2}\right), \quad y_{6}^{\prime}(u)=\alpha_{-}^{2} e^{2 i \phi_{-}} u\left(u-\frac{\eta}{2}\right),
\end{aligned}
$$

$p_{-}, \alpha_{-}$and $\phi_{-}$are the boundary parameters which measure the strength and direction of the boundary field. The reflection matrix $K^{-}(u)$ satisfies the reflection equation (RE)

$$
R_{12}(u-v) K_{1}^{-}(u) R_{21}(u+v) K_{2}^{-}(v)=K_{2}^{-}(v) R_{21}(u+v) K_{1}^{-}(u) R_{12}(u-v) .
$$

The most general off-diagonal boundary reflection at the other side is quantified by the dual reflection matrix

$$
K^{+}(u)=\left.K^{-}(-u-\eta)\right|_{\left(p_{-}, \alpha_{-}, \phi_{-}\right) \rightarrow\left(p_{+},-\alpha_{+}, \phi_{+}\right)},
$$

where $p_{+}, \alpha_{+}$and $\phi_{+}$are the boundary parameters characterizing the strength and direction of the corresponding boundary field. The dual reflection matrix $K^{+}(u)$ satisfies the dual RE

$$
R_{12}(v-u) K_{1}^{+}(u) R_{21}(-u-v-2 \eta) K_{2}^{+}(v)=K_{2}^{+}(v) R_{21}(-u-v-2 \eta) K_{1}^{+}(u) R_{12}(v-u) .
$$

From the $R$-matrix (2), we construct the single row monodromy matrices $T_{0}(u)$ and $\hat{T}_{0}(u)$ as

$$
\begin{aligned}
& T_{0}(u)=R_{0 N}\left(u-\theta_{N}\right) R_{0 N-1}\left(u-\theta_{N-1}\right) \ldots R_{01}\left(u-\theta_{1}\right) \\
& \hat{T}_{0}(u)=R_{10}\left(u+\theta_{1}\right) R_{20}\left(u+\theta_{2}\right) \ldots R_{N 0}\left(u+\theta_{N}\right)
\end{aligned}
$$


where $\left\{\theta_{k}, k=1, \ldots, N\right\}$ are the inhomogeneous parameters, and the subscript 0 means the auxiliary space and $1, \ldots, N$ denote the quantum spaces. The single row monodromy matrices $T_{0}(u)$ and $\hat{T}_{0}(u)$ are the $3 \times 3$ matrices in the auxiliary space $V_{0}$ and their elements act on the quantum space $\mathbf{V}^{\otimes N}$. The transfer matrix of the system reads

$$
t(u)=\operatorname{tr}_{0}\left\{K_{0}^{+}(u) T_{0}(u) K_{0}^{-}(u) \hat{T}_{0}(u)\right\} .
$$

From the QYBE (4), RE (9) and dual RE (11), one can prove that the transfer matrices with different spectral parameters commute with each other, i.e.,

$$
[t(u), t(v)]=0 .
$$

Therefore, $t(u)$ serves as the generating functional of all the conserved quantities, which ensures the integrability of the system. The model Hamiltonian is generated from the transfer matrix $t(u)$ as [19]

$$
\begin{aligned}
H= & \left.\partial_{u}\{\ln t(u)\}\right|_{u=0,\left\{\theta_{k}=0\right\}} \\
= & \frac{1}{\eta} \sum_{k=1}^{N-1}\left[\vec{S}_{k} \cdot \vec{S}_{k+1}-\left(\vec{S}_{k} \cdot \vec{S}_{k+1}\right)^{2}\right] \\
& +\frac{1}{p_{-}^{2}-\frac{1}{4}\left(1+\alpha_{-}^{2}\right) \eta^{2}}\left[2 p_{-}\left(\alpha_{-} \cos \phi_{-} S_{1}^{x}-\alpha_{-} \sin \phi_{-} S_{1}^{y}+S_{1}^{z}\right)-\eta\left(S_{1}^{z}\right)^{2}\right. \\
& \quad-\frac{1}{2} \alpha_{-}^{2} \eta\left[\cos \left(2 \phi_{-}\right)\left[\left(S_{1}^{x}\right)^{2}-\left(S_{1}^{y}\right)^{2}\right]-\left(S_{1}^{z}\right)^{2}\right]-\alpha_{-} \eta \cos \phi_{-}\left[S_{1}^{x} S_{1}^{z}+S_{1}^{z} S_{1}^{x}\right] \\
& \left.+\frac{1}{2} \alpha_{-}^{2} \eta \sin \left(2 \phi_{-}\right)\left[S_{1}^{x} S_{1}^{y}+S_{1}^{y} S_{1}^{x}\right]+\alpha_{-} \eta \sin \phi_{-}\left[S_{1}^{y} S_{1}^{z}+S_{1}^{z} S_{1}^{y}\right]\right] \\
& \quad \frac{1}{p_{+}^{2}-\frac{1}{4}\left(1+\alpha_{+}^{2}\right) \eta^{2}}\left[2 p_{+}\left(\alpha_{+} \cos \phi_{+} S_{N}^{x}-\alpha_{+} \sin \phi_{+} S_{N}^{y}-S_{N}^{z}\right)-\eta\left(S_{N}^{z}\right)^{2}\right. \\
& \quad-\frac{1}{2} \alpha_{+}^{2} \eta\left[\cos \left(2 \phi_{+}\right)\left[\left(S_{N}^{x}\right)^{2}-\left(S_{N}^{y}\right)^{2}\right]-\left(S_{N}^{z}\right)^{2}\right]+\alpha_{+} \eta \cos \phi_{+}\left[S_{N}^{x} S_{N}^{z}+S_{N}^{z} S_{N}^{x}\right] \\
& \left.+\frac{1}{2} \alpha_{+}^{2} \eta \sin \left(2 \phi_{+}\right)\left[S_{N}^{x} S_{N}^{y}+S_{N}^{y} S_{N}^{x}\right]-\alpha_{+} \eta \sin \phi_{+}\left[S_{N}^{y} S_{N}^{z}+S_{N}^{z} S_{N}^{y}\right]\right] \\
& \frac{\eta}{p_{+}^{2}-\frac{1}{4}\left(1+\alpha_{+}^{2}\right) \eta^{2}}+\frac{\eta}{p_{-}^{2}-\frac{1}{4}\left(1+\alpha_{-}^{2}\right) \eta^{2}}+\frac{1}{\eta}\left(3 N+\frac{8}{3}\right)
\end{aligned}
$$

Now, we seek the exact solution of the system (15). Let $|\Psi\rangle$ be an arbitrary eigenstate of $t(u)$ with the eigenvalue $\Lambda(u)$, i.e.,

$$
t(u)|\Psi\rangle=\Lambda(u)|\Psi\rangle .
$$

Using the ODBA method [50] and fusion hierarchy, in the homogeneous limit $\left\{\theta_{k}=0\right\}$, the eigenvalue $\Lambda(u)$ can be expressed as the inhomogeneous $T-Q$ relation,

$$
\begin{aligned}
\Lambda(u) & =-4 u(u+\eta) \Lambda^{\left(\frac{1}{2}, 1\right)}\left(u+\frac{\eta}{2}\right) \Lambda^{\left(\frac{1}{2}, 1\right)}\left(u-\frac{\eta}{2}\right)+4 u(u+\eta) \delta^{(1)}\left(u+\frac{\eta}{2}\right), \\
\Lambda^{\left(\frac{1}{2}, 1\right)}(u) & =a^{(1)}(u) \frac{Q(u-\eta)}{Q(u)}+d^{(1)}(u) \frac{Q(u+\eta)}{Q(u)}+c u(u+\eta) \frac{F^{(1)}(u)}{Q(u)},
\end{aligned}
$$


where

$$
\begin{aligned}
a^{(1)}(u) & =d^{(1)}(-u-\eta) \\
& =-\frac{2 u+2 \eta}{2 u+\eta}\left(\sqrt{1+\alpha_{+}^{2}} u+p_{+}\right)\left(\sqrt{1+\alpha_{-}^{2}} u-p_{-}\right)\left(u+\frac{3 \eta}{2}\right)^{2 N}, \\
F^{(1)}(u) & =\left(u-\frac{\eta}{2}\right)^{2 N}\left(u+\frac{\eta}{2}\right)^{2 N}\left(u+\frac{3 \eta}{2}\right)^{2 N}, \\
\delta^{(1)}(u) & =a^{(1)}(u) d^{(1)}(u-\eta), \\
c & =2\left[\alpha_{-} \alpha_{+} \cos \left(\phi_{+}-\phi_{-}\right)-1+\sqrt{\left(1+\alpha_{-}^{2}\right)\left(1+\alpha_{+}^{2}\right)}\right], \\
Q(u) & =\prod_{k=1}^{2 N}\left(u-u_{k}\right)\left(u+u_{k}+\eta\right)=Q(-u-\eta),
\end{aligned}
$$

and the $2 N$ parameters $\left\{u_{k} \mid k=1, \ldots, 2 N\right\}$ in $Q$-function (23) are the Bethe roots. The singularity of eigenvalue $\Lambda(u)$ requires that the Bethe roots should satisfy the BAEs

$$
a^{(1)}\left(u_{k}\right) Q\left(u_{k}-\eta\right)+d^{(1)}\left(u_{k}\right) Q\left(u_{k}+\eta\right)+c u_{k}\left(u_{k}+\eta\right) F^{(1)}\left(u_{k}\right)=0, \quad k=1, \ldots, 2 N .
$$

The eigenvalue of Hamiltonian (15) reads

$$
E=\sum_{k=1}^{2 N} \frac{4 \eta}{\left(u_{k}+\frac{3 \eta}{2}\right)\left(u_{k}-\frac{\eta}{2}\right)}+\frac{1}{\eta} 3 N+\frac{1}{\eta} E_{0},
$$

where $\left\{u_{k}\right\}$ should satisfy the BAEs (24) and

$$
E_{0}=\frac{8}{3}+\frac{2 \sqrt{1+\alpha_{+}^{2}} p_{+} \eta}{p_{+}^{2}-\frac{\eta^{2}}{4}\left(1+\alpha_{+}^{2}\right)}-\frac{2 \sqrt{1+\alpha_{-}^{2}} p_{-} \eta}{p_{-}^{2}-\frac{\eta^{2}}{4}\left(1+\alpha_{-}^{2}\right)} .
$$

Some remarks are in order. If the non-diagonal boundary parameters are $\alpha_{+}=\alpha_{-}=0$, or $\alpha_{+}=-\alpha_{-} \neq 0$ and $\phi_{-}=\phi_{+}$(which corresponds to the parallel boundary fields case), the parameter $c$ in Eq.(22) becomes zero and the corresponding $T-Q$ relation (18) is naturally reduced to the conventional diagonal one [30] obtained by the algebraic Bethe Ansatz. ${ }^{2}$ For the other case with unparallel boundary fields, the parameter $c$ does not vanish. Thus the corresponding $T-Q$ relation has to include a non-vanishing inhomogeneous term for any finite $N$.

\section{Finite size scaling behavior}

The present BAEs (24) are inhomogeneous, thus it is very hard to investigate the thermodynamic properties of the system by using the traditional thermodynamic Bethe ansatz. In order to overcome this difficulty, we first analyze the contribution of inhomogeneous term in the $T-Q$ relation (18).

Define the reduced $T-Q$ relation as

$$
\begin{aligned}
\Lambda_{h o m}(u) & =-4 u(u+\eta) \Lambda_{h o m}^{\left(\frac{1}{2}, 1\right)}\left(u+\frac{\eta}{2}\right) \Lambda_{h o m}^{\left(\frac{1}{2}, 1\right)}\left(u-\frac{\eta}{2}\right)+4 u(u+\eta) \delta^{(1)}\left(u+\frac{\eta}{2}\right), \\
\Lambda_{h o m}^{\left(\frac{1}{2}, 1\right)}(u) & =a^{(1)}(u) \frac{Q(u-\eta)}{Q(u)}+d^{(1)}(u) \frac{Q(u+\eta)}{Q(u)} .
\end{aligned}
$$

\footnotetext{
${ }^{2}$ If the non-diagonal boundary parameters satisfy the condition $\alpha_{+}=\alpha_{-} \neq 0,\left|\phi_{-}-\phi_{+}\right|=\pi$ (which corresponds to the antiparallel boundary fields case), the parameter $c$ in Eq.(22) also becomes zero and the corresponding $T-Q$ relation naturally degenerates into the conventional diagonal one.
} 
It should be emphasized that although the non-diagonal boundary parameters $\left\{p_{ \pm}, \alpha_{ \pm}\right\}$except $\phi_{ \pm}$are included in the above reduced $T-Q$ relation (28), the $\Lambda_{h o m}(u)$ is not the eigenvalue $\Lambda(u)$ for any finite $N$ but rather that of the transfer matrix with parallel boundary fields of the same strength. In the limit $N \rightarrow \infty$ it will give, however, the correct boundary energy (see the following parts of the paper). From the singularity analysis of the reduced $T-Q$ relation (28), we obtain the following reduced BAEs

$$
\frac{\frac{i}{2}-\mu_{k}}{\frac{i}{2}+\mu_{k}} \frac{p i-\mu_{k}}{p i+\mu_{k}} \frac{q i-\mu_{k}}{q i+\mu_{k}}\left(\frac{i-\mu_{k}}{i+\mu_{k}}\right)^{2 N}=\prod_{l=1}^{M} \frac{i-\left(\mu_{k}-\mu_{l}\right)}{i+\left(\mu_{k}-\mu_{l}\right)} \frac{i-\left(\mu_{k}+\mu_{l}\right)}{i+\left(\mu_{k}+\mu_{l}\right)}, k=1, \ldots, M,
$$

where $M=1, \ldots, 2 N$ and we have put $\eta=1, \mu_{k}=-i u_{k}-\frac{i}{2}, p=\frac{p_{+}}{\sqrt{1+\alpha_{+}^{2}}}-\frac{1}{2}$ and $q=-\frac{p_{-}}{\sqrt{1+\alpha_{-}^{2}}}-\frac{1}{2}$ for convenience. From the $\Lambda_{\text {hom }}(u)$ given by Eq.(27), we obtain the reduced energy which is defined as

$$
E_{\text {hom }}=\left.\partial_{u}\left\{\ln \Lambda_{\text {hom }}(u)\right\}\right|_{u=0}=-\sum_{k=1}^{M} \frac{4}{\mu_{k}^{2}+1}+3 N+E_{0} .
$$

Solving the reduced BAEs (29), we could obtain the values of reduced Bethe roots $\left\{\mu_{k}\right\}$. Substituting the Bethe roots into Eq.(30), we obtain the values of $E_{h o m}$.

Let us focus on the ground state. The reduced ground state energy can be calculated by the reduced BAEs (29). It is well-known that the even $N$ and odd $N$ give the same physical properties in the thermodynamic limit. Thus we set $N$ as even. At the ground state, the number of Bethe roots in the reduced BAEs (29) is $M=N$. For simplicity, we choose the boundary parameters as $p>0$ and $q \neq 0,-1$. We should note that at the points of $q=0,-1$, the boundary field is divergent due to the present parameterization of the Hamiltonian (15). The distribution of reduced Bethe roots at the ground state in the thermodynamic limit is shown in Figure 1. We see that the Bethe roots can be divided into six different regimes in the $p-q$ plane.

1) In the regime I, where $p \geq 1 / 2, q<-1,-1 / 2 \leq q<0$ or $q \geq 1 / 2$, all the Bethe roots form 2-strings, i.e., $\mu_{k}=\lambda_{k} \pm \frac{i}{2}+\mathcal{O}\left(e^{-\delta N}\right)$, where $\lambda_{k}$ denotes the position of 2 -string in the real axis, $\delta$ is a small positive number and $\mathcal{O}\left(e^{-\delta N}\right)$ means the finite size correction.

2) In the regime II, where $p<1 / 2, q<-1,-1 / 2 \leq q<0$ or $q \geq 1 / 2$, besides $N-2$ 2 -strings, there are two boundary strings, i.e., $p i$ and $(p-1) i$. The boundary strings mean the pure imaginary Bethe roots which are related with the boundary parameters $p$ and $q$ [63].

3 ) In the regime III, where $p \geq 1 / 2$ and $0<q<1 / 2$, besides $N-22$-strings, there are two boundary strings, $q i$ and $(q-1) i$.

4) In the regime IV, where $0<p<1 / 2$ and $0<q<1 / 2$, besides $N-42$-strings, there are four boundary strings, $p i,(p-1) i, q i$ and $(q-1) i$.

5) In the regime $\mathrm{V}$, where $p \geq 1 / 2$ and $-1<q<-1 / 2$, besides $N-22$-strings, only the boundary string $q i$ survives and one real Bethe root $\lambda_{0}$ appears which is caused by the rearrangement of Fermi sea.

6) In the regime VI, where $0<p<1 / 2$ and $-1<q<-1 / 2$, besides $N-42$-strings, there are three boundary strings $q i,(q-1) i, p i$ and one real root $\lambda_{0}$.

Because the Bethe roots are different in the different regimes of boundary parameters, we shall discuss them separately. In the regime I, where all the Bethe roots are the 2-strings. Substituting the 2-string solutions into the reduced BAEs (29), omitting the exponentially 


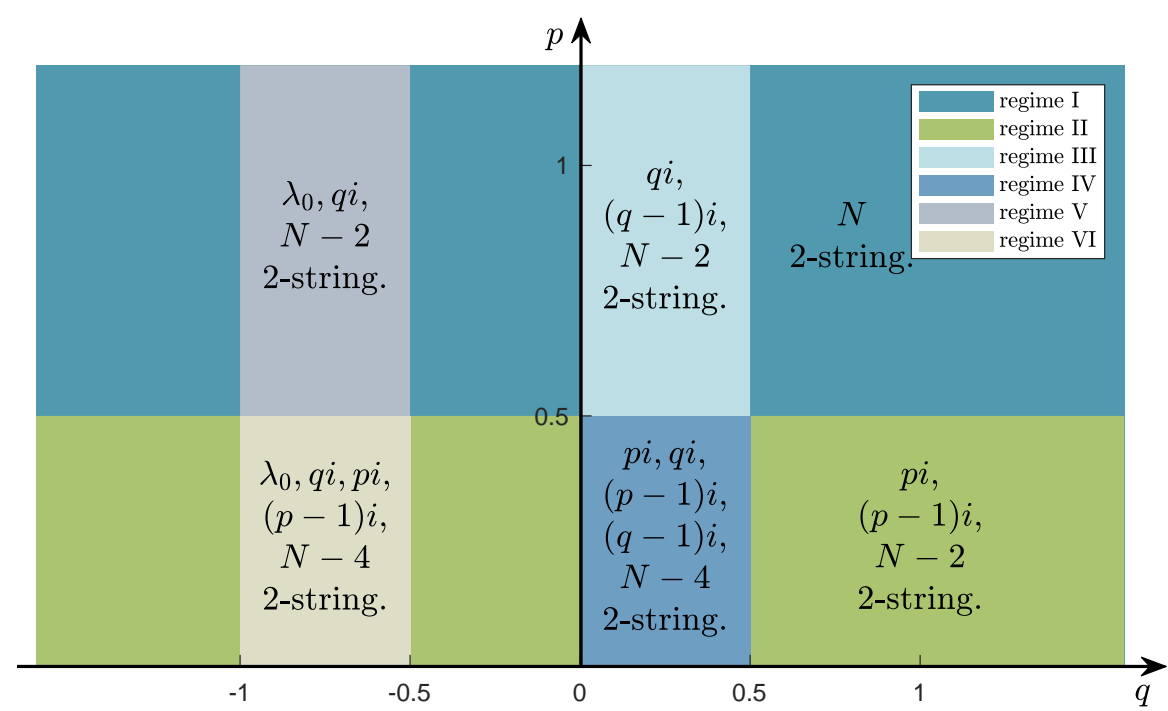

Figure 1: The distribution of reduced Bethe roots at the ground states with different boundary parameters $p$ and $q$.

minor corrections and taking the product of all the string solutions, we readily obtain

$$
\begin{aligned}
& -\frac{i-\lambda_{j}}{i+\lambda_{j}} \frac{\left(p-\frac{1}{2}\right) i-\lambda_{j}}{\left(p-\frac{1}{2}\right) i+\lambda_{j}} \frac{\left(p+\frac{1}{2}\right) i-\lambda_{j}}{\left(p+\frac{1}{2}\right) i+\lambda_{j}} \frac{\left(q-\frac{1}{2}\right) i-\lambda_{j}}{\left(q-\frac{1}{2}\right) i+\lambda_{j}} \frac{\left(q+\frac{1}{2}\right) i-\lambda_{j}}{\left(q+\frac{1}{2}\right) i+\lambda_{j}} \\
& \quad \times\left(\frac{\frac{1}{2} i-\lambda_{j}}{\frac{1}{2} i+\lambda_{j}} \frac{\frac{3}{2} i-\lambda_{j}}{\frac{3}{2} i+\lambda_{j}}\right)^{2 N}=\prod_{l=1}^{M_{1}}\left[\frac{i-\left(\lambda_{j}-\lambda_{l}\right)}{i+\left(\lambda_{j}-\lambda_{l}\right)}\right]^{2}\left[\frac{i-\left(\lambda_{j}+\lambda_{l}\right)}{i+\left(\lambda_{j}+\lambda_{l}\right)}\right]^{2} \\
& \quad \times \frac{2 i-\left(\lambda_{j}-\lambda_{l}\right)}{2 i+\left(\lambda_{j}-\lambda_{l}\right)} \frac{2 i-\left(\lambda_{j}+\lambda_{l}\right)}{2 i+\left(\lambda_{j}+\lambda_{l}\right)}, \quad j=1, \ldots, M_{1} .
\end{aligned}
$$

Taking the logarithm of above Eq.(31), we obtain

$$
2 \pi I_{j}=W\left(\lambda_{j} ; M_{1}\right)+\theta_{2 p-1}\left(\lambda_{j}\right)+\theta_{2 p+1}\left(\lambda_{j}\right)+\theta_{2 q-1}\left(\lambda_{j}\right)+\theta_{2 q+1}\left(\lambda_{j}\right), j=1, \ldots, M_{1},
$$

where

$$
\begin{aligned}
W\left(\lambda_{j} ; M_{1}\right)= & \theta_{2}\left(\lambda_{j}\right)+2 N\left[\theta_{1}\left(\lambda_{j}\right)+\theta_{3}\left(\lambda_{j}\right)\right] \\
& -\sum_{l=1}^{M_{1}}\left[2 \theta_{2}\left(\lambda_{j}-\lambda_{l}\right)+2 \theta_{2}\left(\lambda_{j}+\lambda_{l}\right)+\theta_{4}\left(\lambda_{j}-\lambda_{l}\right)+\theta_{4}\left(\lambda_{j}+\lambda_{l}\right)\right],
\end{aligned}
$$

$I_{j}$ is the quantum number, $\theta_{n}(x)=2 \arctan (2 x / n)$ and $M_{1}=N / 2$. The ground state is characterized by the set of quantum numbers

$$
\left\{I_{j}\right\}=\left\{1,2, \ldots, M_{1}\right\} .
$$

Solving the reduced BAEs (32) and substituting the values of Bethe roots into Eq.(30), we obtain the reduced ground state energy as

$$
E_{\text {hom }}=-2 \sum_{j=1}^{M_{1}} \frac{1}{\lambda_{j}^{2}+\frac{1}{4}}+\frac{3}{\lambda_{j}^{2}+\frac{9}{4}}+3 N+E_{0} \equiv G\left(\lambda_{j} ; M_{1}\right) .
$$



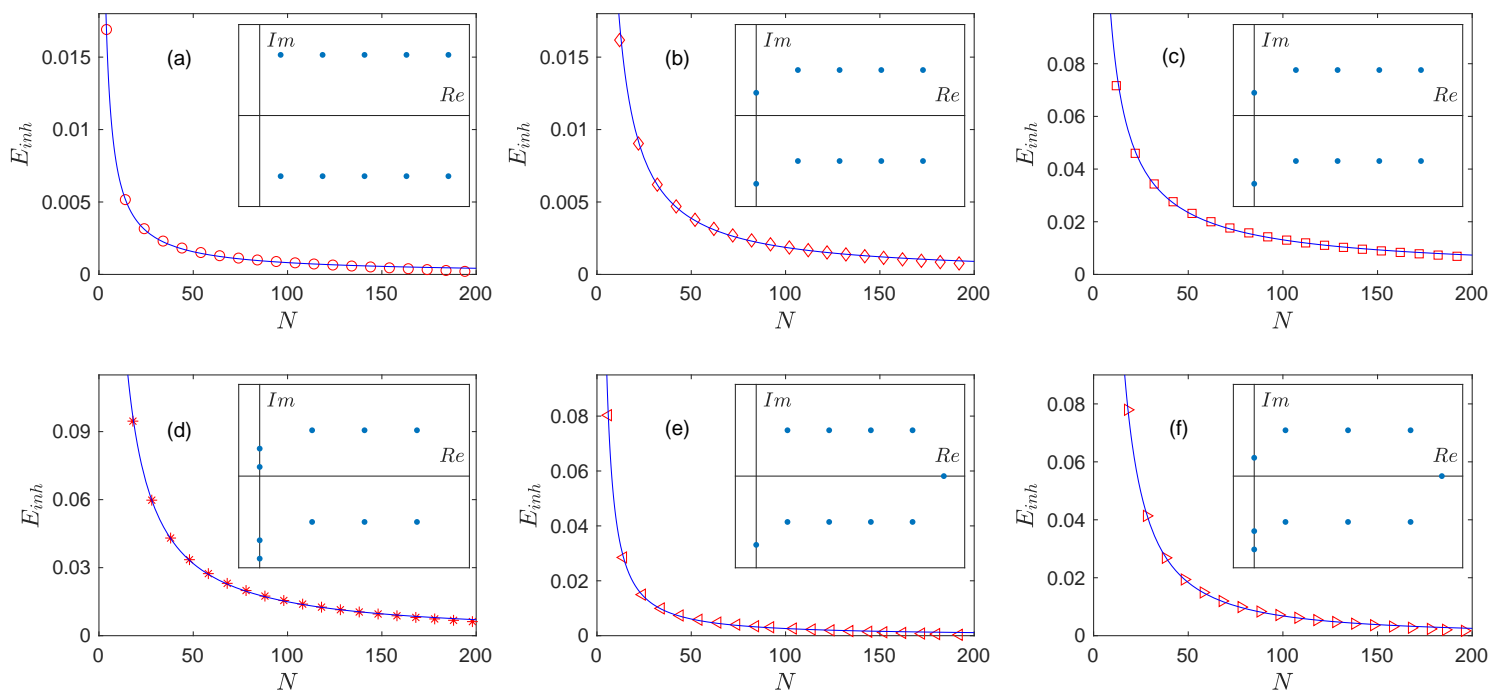

Figure 2: The values of $E_{\text {inh }}$ versus the system size $N$. The data can be fitted as $E_{i n h}=\gamma N^{\beta}$. Due to the fact $\beta<0$, when the size of system $N \rightarrow \infty$, the contribution of the inhomogeneous term tends to zero. Here (a) $p=1.1370, q=-1.0821, \gamma=0.06203$ and $\beta=-0.9407$ in regime I; (b) $p=0.3263, q=-1.8931, \gamma=0.2371$ and $\beta=-1.052$ in regime II; (c) $p=0.2428, q=2.3735, \gamma=0.6236$ and $\beta=-0.8384$ in regime III; (d) $p=0.4453, q=0.3789, \gamma=2.234$ and $\beta=-1.087$ in regime IV; (e) $p=0.8410, q=-0.6990, \gamma=0.715$ and $\beta=-1.219$ in regime V; (f) $p=0.3971, q=-0.7985, \gamma=4.912$ and $\beta=-1.429$ in regime VI. The insets show the distribution of Bethe roots with $N=10$.

Now, we are ready to characterize the contribution of inhomogeneous term in the $T-Q$ relation (18) at the ground state by the quantity

$$
E_{\text {inh }}=E_{\text {hom }}-E_{g},
$$

where $E_{\text {hom }}$ is the reduced ground state energy given by (35) and $E_{g}$ is the actual ground state energy (25) of the Hamiltonian (15). The ground state energy $E_{g}$ can be obtained by two methods. One is solving the inhomogeneous BAEs (24) directly and the other is DMRG [56-58]. We have checked that the ground state energy $E$ obtained by these two methods are the same.

In Figure 2(a), we give the values of $E_{i n h}$ versus the system size $N$ in the regime I. The red circles are the data calculated from Eq.(36) and the blue solid line is the fitted curve. From the fitted curve, we find that $E_{\text {inh }}$ and $N$ satisfy the power law relation $E_{i n h}=\gamma N^{\beta}$. Due to the fact that $\beta<0$, the value of $E_{\text {inh }}$ tends to zero when the system size $N$ tends to infinity. Therefore, in the thermodynamic limit, the inhomogeneous term in the $T-Q$ relation (18) can be neglected at the ground state and $E_{h o m}=E_{g}$. The inset shows the distribution of Bethe roots with $N=10$.

In the regime II, substituting the $N-2$ 2-strings, two boundary strings $\mu_{M-1}=p i$ and $\mu_{M}=(p-1) i$ into the reduced BAEs (29) and taking the logarithm, we have

$$
\begin{array}{r}
2 \pi I_{j}=W\left(\lambda_{j} ; M_{2}\right)+\theta_{2 q-1}\left(\lambda_{j}\right)+\theta_{2 q+1}\left(\lambda_{j}\right)-\theta_{1-2 p}\left(\lambda_{j}\right)-\theta_{2 p+1}\left(\lambda_{j}\right) \\
-\theta_{3+2 p}\left(\lambda_{j}\right)-\theta_{5-2 p}\left(\lambda_{j}\right)-2 \theta_{3-2 p}\left(\lambda_{j}\right), \quad j=1,2, \ldots, M_{2},
\end{array}
$$


where $W\left(\lambda_{j} ; M_{2}\right)$ is given by Eq. (33) with the replacing of $M_{1}$ by $M_{2}, M_{2}=N / 2-1$ and the quantum numbers are

$$
\left\{I_{j}\right\}=\left\{1,2, \ldots, M_{2}\right\} .
$$

The corresponding reduced ground state energy reads

$$
E_{\text {hom }}=G\left(\lambda_{j} ; M_{2}\right)+\frac{4}{p^{2}-1}+\frac{4}{(p-1)^{2}-1},
$$

where $G\left(\lambda_{j} ; M_{2}\right)$ is given by Eq.(35) with the replacing of $M_{1}$ by $M_{2}$.

The procedure in the regime III is similar and reduced ground state energy is

$$
E_{\text {hom }}=G\left(\lambda_{j} ; M_{2}\right)+\frac{4}{q^{2}-1}+\frac{4}{(q-1)^{2}-1} .
$$

In the regime IV, substituting the string solutions including four boundary strings into Eq.(29) and taking the logarithm, we have

$$
\begin{aligned}
& 2 \pi I_{j}=W\left(\lambda_{j} ; M_{3}\right)-\theta_{1-2 p}\left(\lambda_{j}\right)-\theta_{2 p+1}\left(\lambda_{j}\right)-\theta_{3+2 p}\left(\lambda_{j}\right)-\theta_{5-2 p}\left(\lambda_{j}\right)-2 \theta_{3-2 p}\left(\lambda_{j}\right) \\
& -\theta_{1-2 q}\left(\lambda_{j}\right)-\theta_{2 q+1}\left(\lambda_{j}\right)-\theta_{3+2 q}\left(\lambda_{j}\right)-\theta_{5-2 q}\left(\lambda_{j}\right)-2 \theta_{3-2 q}\left(\lambda_{j}\right), \quad j=1,2, \ldots, M_{3},
\end{aligned}
$$

where $M_{3}=N / 2-2$ and the quantum numbers are

$$
\left\{I_{j}\right\}=\left\{1,2, \ldots, M_{3}\right\} .
$$

The reduced ground state energy is

$$
E_{\text {hom }}=G\left(\lambda_{j} ; M_{3}\right)+\frac{4}{p^{2}-1}+\frac{4}{(p-1)^{2}-1}+\frac{4}{q^{2}-1}+\frac{4}{(q-1)^{2}-1} .
$$

In the regime $\mathrm{V}$, the logarithm form of the BAEs are

$$
\begin{array}{r}
2 \pi I_{j}=W\left(\lambda_{j} ; M_{4}\right)+\theta_{2 p-1}\left(\lambda_{j}\right)+\theta_{2 p+1}\left(\lambda_{j}\right)-\theta_{3+2 q}\left(\lambda_{j}\right)-\theta_{3-2 q}\left(\lambda_{j}\right)-2 \theta_{1-2 q}\left(\lambda_{j}\right) \\
-\theta_{1}\left(\lambda_{j}-\lambda_{0}\right)-\theta_{1}\left(\lambda_{j}+\lambda_{0}\right)-\theta_{3}\left(\lambda_{j}-\lambda_{0}\right)-\theta_{3}\left(\lambda_{j}+\lambda_{0}\right), \quad j=1,2, \ldots, M_{4},
\end{array}
$$

where $M_{4}=N / 2-1$ and the quantum numbers are $\left\{I_{j}\right\}=\left\{1,2, \ldots, M_{4}\right\}$. We shall note that the quantum number corresponding to the real Bethe root $\lambda_{0}$ is 0 . The reduced ground state energy reads

$$
E_{\text {hom }}=G\left(\lambda_{j} ; M_{4}\right)+\frac{4}{q^{2}-1}-\frac{4}{\lambda_{0}^{2}+1} .
$$

Similarly, the reduced ground state energy in the regime VI is

$$
E_{\text {hom }}=G\left(\lambda_{j} ; M_{5}\right)+\frac{4}{p^{2}-1}+\frac{4}{(p-1)^{2}-1}+\frac{4}{q^{2}-1}-\frac{4}{\lambda_{0}^{2}+1},
$$

where $M_{5}=N / 2-2$.

Substituting the reduced ground state energies in different regimes into Eq.(36), we obtain the values of $E_{i n h}$, which are shown in Figures 2(b)-(f). According to the finite size scaling analysis, we see that the inhomogeneous term indeed can be neglected at the ground state in the thermodynamic limit. Due to the existence of inhomogeneous term in BAEs.(24), it is hard to analytically calculate the finite size correction for the present off-diagonal boundary reflections along the lines given in references [64-66]. We shall note that the diagonal case is tractable along the lines of A. Klümper et al. [65] and J. Suzuki [66]. The $\mathcal{O}\left(N^{1}\right)$ bulk term and the $\mathcal{O}\left(N^{0}\right)$ boundary term for the ground state energy do not depend on the orientations of the boundary fields. The true finite size correction terms are probably of order $\mathcal{O}\left(N^{-1}\right)$ and are out of reach for the inhomogeneous/off-diagonal case. Due to higher order correction terms, the effective exponents $\beta$ determined in the paper differ from -1 . 


\section{Boundary energy}

In this section, we study the physical effects induced by the boundary magnetic fields and compute the boundary energy in the thermodynamic limit [18, 35, 67-69]. As mentioned above, we can calculate the boundary energy based on the string hypothesis of the reduced BAEs (29), then the numerical analysis allows us to obtain the boundary energy induced by the boundary fields.

The values of Bethe roots at the ground state are determined by the quantum numbers $\left\{I_{j}\right\}$. Thus we define the counting function as $Z\left(\lambda_{j}\right)=\frac{I_{j}}{2 N}$. In the thermodynamic limit, the Bethe roots can take the continuous values and we have $Z\left(\lambda_{j}\right) \rightarrow Z(u)$. Taking the derivative of $Z(u)$ with respect to $u$, we obtain

$$
\frac{d Z(u)}{d u}=\rho(u)+\rho^{h}(u),
$$

where $\rho(u)$ is the density of Bethe roots and $\rho^{h}(u)$ means the density of holes in the real axis. Again, the distribution of Bethe roots in different regimes are different. We should consider them separately. In regime I, from the BAEs (32) with the constraint $N \rightarrow \infty$ and using Eq.(47), we obtain the density of states as

$$
\begin{aligned}
\rho(u)= & \frac{d Z(u)}{d u}-\frac{1}{2 N}\left[\rho^{h}(u)+\delta(u)\right] \\
= & a_{1}(u)+a_{3}(u)+\frac{1}{2 N}\left[a_{2}(u)+a_{2 p-1}(u)+a_{2 p+1}(u)+a_{2 q-1}(u)+a_{2 q+1}(u)\right] \\
& -\frac{1}{2 N}\left[\rho^{h}(u)+\delta(u)\right]-\int_{-\infty}^{\infty}\left[2 a_{2}(u-v)+a_{4}(u+v)\right] \rho(v) d v,
\end{aligned}
$$

where

$$
\begin{aligned}
& a_{n}(u)=\frac{1}{2 \pi} \frac{n}{u^{2}+\frac{n^{2}}{4}}, \\
& \rho^{h}(u)=\frac{1}{2 N}\left[\delta\left(u-\lambda_{1}^{h}\right)+\delta\left(u+\lambda_{1}^{h}\right)+\delta\left(u-\lambda_{2}^{h}\right)+\delta\left(u+\lambda_{2}^{h}\right)\right] .
\end{aligned}
$$

We should note that the presence of delta-function in Eq.(48) is due to that $\lambda_{j}=0$ is the solution of BAEs (32), which should be excluded because it makes the wavefunction vanish identically [70]. Note that two holes $\lambda_{1}^{h}$ and $\lambda_{2}^{h}$ are introduced to ensure the magnetization satisfying

$$
\frac{M}{N}=2 \int_{-\infty}^{\infty} \rho(u) d u=1 .
$$

Thus the holes are located at the infinities in the real axis.

With the help of Fourier transformation

$$
\tilde{F}(\omega)=\int_{-\infty}^{\infty} e^{i \omega u} F(u) d u, \quad F(u)=\frac{1}{2 \pi} \int_{-\infty}^{\infty} e^{-i \omega u} \tilde{F}(\omega) d \omega,
$$

from Eq.(48), we obtain

$$
\tilde{\rho}(\omega)=\tilde{\rho}_{g}(\omega)+\tilde{\rho}_{0}(\omega)+\tilde{\rho}_{1}(\omega)+\tilde{\rho}_{2}(\omega),
$$


where

$$
\begin{aligned}
& \tilde{a}_{n}(\omega)=e^{-\frac{n|\omega|}{2}}, \quad \tilde{\rho}_{g}(\omega)=\frac{\tilde{a}_{1}(\omega)+\tilde{a}_{3}(\omega)}{1+2 \tilde{a}_{2}(\omega)+\tilde{a}_{4}(\omega)}, \quad \tilde{\rho}_{0}(\omega)=\frac{1}{2 N} \frac{\tilde{a}_{2}(\omega)-1}{1+2 \tilde{a}_{2}(\omega)+\tilde{a}_{4}(\omega)}, \\
& \tilde{\rho}_{1}(\omega)=\left\{\begin{array}{lc}
\frac{1}{2 N} \frac{\tilde{a}_{2 p+1}(\omega)-\tilde{a}_{1-2 p}(\omega)}{1+2 \tilde{a}_{2}(\omega)+\tilde{a}_{4}(\omega)}, & 0<p<\frac{1}{2}, \\
\frac{1}{2 N} \frac{\tilde{a}_{2 p-1}(\omega)+\tilde{a}_{2 p+1}(\omega)}{1+2 \tilde{a}_{2}(\omega)+\tilde{a}_{4}(\omega)}, & p>\frac{1}{2},
\end{array}\right. \\
& \tilde{\rho}_{2}(\omega)=\left\{\begin{array}{lc}
-\frac{1}{2 N} \frac{\tilde{a}_{1-2 q}(\omega)+\tilde{a}_{-2 q-1}(\omega)}{1+2 \tilde{a}_{2}(\omega)+\tilde{a}_{4}(\omega)}, & q<-\frac{1}{2}, \\
\frac{1}{2 N} \frac{\tilde{a}_{2 q+1}(\omega)-\tilde{a}_{1-2 q}(\omega)}{1+2 \tilde{a}_{2}(\omega)+\tilde{a}_{4}(\omega)}, & -\frac{1}{2}<q<\frac{1}{2}, \\
\frac{1}{2 N} \frac{\tilde{a}_{2 q-1}(\omega)+\tilde{a}_{2 q+1}(\omega)}{1+2 \tilde{a}_{2}(\omega)+\tilde{a}_{4}(\omega)}, & q>\frac{1}{2} .
\end{array}\right.
\end{aligned}
$$

Then the ground state energy (35) can be expressed as

$$
E_{g}=-2 N \int_{-\infty}^{\infty}\left[\tilde{a}_{1}(\omega)+\tilde{a}_{3}(\omega)\right] \tilde{\rho}(\omega) d \omega+3 N+E_{0}=N e_{g}+e_{s},
$$

where $e_{g}$ is the ground state energy density which is the same as that for the periodic boundary condition [9],

$$
e_{g}=-2 \int_{-\infty}^{\infty} \frac{\left[\tilde{a}_{1}(\omega)+\tilde{a}_{3}(\omega)\right]^{2}}{1+2 \tilde{a}_{2}(\omega)+\tilde{a}_{4}(\omega)} d \omega+3=-1,
$$

and $e_{s}$ is boundary energy

$$
\begin{aligned}
& e_{s}=2 \pi-4+E_{0}+e_{1}+e_{2}, \\
& e_{1}= \begin{cases}-\int_{-\infty}^{\infty}\left[\tilde{a}_{1}(\omega)+\tilde{a}_{3}(\omega)\right] \frac{\tilde{a}_{2 p-1}(\omega)+\tilde{a}_{2 p+1}(\omega)}{1+2 \tilde{a}_{2}(\omega)+\tilde{a}_{4}(\omega)} d \omega, & p>\frac{1}{2}, \\
-\int_{-\infty}^{\infty}\left[\tilde{a}_{1}(\omega)+\tilde{a}_{3}(\omega)\right] \frac{\tilde{a}_{2 p+1}(\omega)-\tilde{a}_{1-2 p}(\omega)}{1+2 \tilde{a}_{2}(\omega)+\tilde{a}_{4}(\omega)} d \omega, & 0<p<\frac{1}{2},\end{cases} \\
& e_{2}=\left\{\begin{array}{lr}
\int_{-\infty}^{\infty}\left[\tilde{a}_{1}(\omega)+\tilde{a}_{3}(\omega)\right] \frac{\tilde{a}_{-2 q-1}(\omega)+\tilde{a}_{1-2 q}(\omega)}{1+2 \tilde{a}_{2}(\omega)+\tilde{a}_{4}(\omega)} d \omega, & q<-\frac{1}{2}, \\
-\int_{-\infty}^{\infty}\left[\tilde{a}_{1}(\omega)+\tilde{a}_{3}(\omega)\right] \frac{\tilde{a}_{2 q+1}(\omega)-\tilde{a}_{1-2 q}(\omega)}{1+2 \tilde{a}_{2}(\omega)+\tilde{a}_{4}(\omega)} d \omega, & -\frac{1}{2}<q<\frac{1}{2}, \\
-\int_{-\infty}^{\infty}\left[\tilde{a}_{1}(\omega)+\tilde{a}_{3}(\omega)\right] \frac{\tilde{a}_{2 q-1}(\omega)+\tilde{a}_{2 q+1}(\omega)}{1+2 \tilde{a}_{2}(\omega)+\tilde{a}_{4}(\omega)} d \omega, & q>\frac{1}{2} .
\end{array}\right.
\end{aligned}
$$

Now, we consider the regime II. The boundary strings $p i$ and $(p-1) i$ can give rise to the rearrangement of Bethe roots in Fermi sea. From BAEs (37), the density of states $\rho_{p}(u)$ is obtained as

$$
\begin{aligned}
\rho_{p}(u)= & a_{1}(u)+a_{3}(u)-\int_{-\infty}^{\infty}\left[2 a_{2}(u-v)+a_{4}(u-v)\right] \rho_{p}(v) d v \\
& +\frac{1}{2 N}\left[a_{2}(u)-a_{1-2 p}(u)+a_{2 p+1}(u)+a_{2 q-1}(u)+a_{2 q+1}(u)-\delta(u)\right] \\
& -\frac{1}{2 N}\left[2 a_{2 p+1}(u)+2 a_{3-2 p}(u)+a_{3+2 p}(u)+a_{5-2 p}(u)\right] .
\end{aligned}
$$


In order to show that there exist the stable boundary bound states, we denote the deviation between $\rho_{p}(u)$ and $\rho(u)$ as $\delta \rho_{p}(u)=\rho_{p}(u)-\rho(u)$. From Eqs. (48) and (59), we obtain

$$
\begin{aligned}
\delta \rho_{p}(u)= & -\frac{1}{2 N}\left[2 a_{2 p+1}(u)+2 a_{3-2 p}(u)+a_{3+2 p}(u)+a_{5-2 p}(u)\right] \\
& -\int_{-\infty}^{\infty}\left[2 a_{2}(u-v)+a_{4}(u-v)\right] \delta \rho_{p}(v) d v .
\end{aligned}
$$

Taking the Fourier transformation of Eq.(60), we have

$$
\delta \tilde{\rho}_{p}(\omega)=-\frac{1}{2 N} \frac{2 \tilde{a}_{2 p+1}(\omega)+2 \tilde{a}_{3-2 p}(\omega)+\tilde{a}_{3+2 p}(\omega)+\tilde{a}_{5-2 p}(\omega)}{1+2 \tilde{a}_{2}(\omega)+\tilde{a}_{4}(\omega)} .
$$

The energy deviation $\delta e_{p}$ induced by the density deviation $\delta \tilde{\rho}_{p}(\omega)$ can be expressed as

$$
\begin{aligned}
\delta e_{p} & =-2 N \int_{-\infty}^{\infty}\left[\tilde{a}_{1}(\omega)+\tilde{a}_{3}(\omega)\right] \delta \tilde{\rho}_{p}(\omega) d \omega+\frac{4}{p^{2}-1}+\frac{4}{(p-1)^{2}-1} \\
& =2 \int_{0}^{\infty} \frac{e^{-(p+1) \omega}}{1+e^{-\omega}} d \omega+2 \int_{0}^{\infty} \frac{e^{-(2-p) \omega}}{1+e^{-\omega}} d \omega+\frac{2}{p(p-1)}<0 .
\end{aligned}
$$

Because of $\delta e_{p}<0$, the boundary strings are stable. Then we conclude that in this regime, the ground state energy of the system is $E_{g}=N e_{g}+e_{s}+\delta e_{p}$. The total spin along the z-direction is $S_{z}=-\int_{-\infty}^{\infty} \delta \rho_{p}(u) d u=3 / 4$.

Next, we consider the regime III where boundary strings are $q i$ and $(q-1) i$. Similarly, the energy deviation $\delta e_{q}$ between this case and that without boundary strings is

$$
\begin{aligned}
\delta e_{q} & =-2 N \int_{-\infty}^{\infty}\left[\tilde{a}_{1}(\omega)+\tilde{a}_{3}(\omega)\right] \delta \tilde{\rho}_{q}(\omega) d \omega+\frac{4}{q^{2}-1}+\frac{4}{(q-1)^{2}-1} \\
& =2 \int_{0}^{\infty} \frac{e^{-(q+1) \omega}}{1+e^{-\omega}} d \omega+2 \int_{0}^{\infty} \frac{e^{-(2-q) \omega}}{1+e^{-\omega}} d \omega+\frac{2}{q(q-1)}<0 .
\end{aligned}
$$

Due to the fact $\delta e_{q}<0$, we know that the ground state energy is $E_{g}=N e_{g}+e_{s}+\delta e_{q}$ and the total spin along the $z$-direction is $S_{z}=3 / 4$.

In the regime IV, we combine the results (62) and (63), and conclude that the ground state energy with boundary strings $p i,(p-1) i$, qi and $(q-1) i$ equals to $E_{g}=N e_{g}+e_{s}+\delta e_{p}+\delta e_{q}$.

Then, we consider the regime $\mathrm{V}$ where besides the $N-2$ 2-string, there also exist one real Bethe root $\lambda_{0}$ and a single boundary string $q i$. Taking the thermodynamic limit of BAEs (44), we obtain the density of states $\rho_{\lambda q}(u)$ as

$$
\begin{aligned}
\rho_{\lambda q}(u)= & a_{1}(u)+a_{3}(u)-\frac{1}{2 N}\left[a_{1}\left(u-\lambda_{0}\right)+a_{1}\left(u+\lambda_{0}\right)+a_{3}\left(u-\lambda_{0}\right)+a_{3}\left(u+\lambda_{0}\right)\right] \\
& +\frac{1}{2 N}\left[a_{2}(u)+a_{2 p-1}(u)+a_{2 p+1}(u)-2 a_{1-2 q}(u)-a_{3+2 q}(u)-a_{3-2 q}(u)-\delta(u)\right] \\
& -\int_{-\infty}^{\infty}\left[2 a_{2}(u-v)+a_{4}(u-v)\right] \rho_{\lambda q}(v) d v .
\end{aligned}
$$

Denote the deviation between $\rho_{\lambda q}(u)$ and $\rho(u)$ as $\delta \rho_{\lambda q}(u)=\rho_{\lambda q}(u)-\rho(u)$. From Eqs.(48) and (64), the value of $\delta \rho_{\lambda q}(u)$ reads

$$
\begin{aligned}
\delta \rho_{\lambda q}(u)= & -\frac{1}{2 N}\left[a_{1}\left(u-\lambda_{0}\right)+a_{1}\left(u+\lambda_{0}\right)+a_{3}\left(u-\lambda_{0}\right)+a_{3}\left(u+\lambda_{0}\right)\right] \\
& -\frac{1}{2 N}\left[a_{1-2 q}(u)-a_{-1-2 q}(u)+a_{3-2 q}(u)+a_{3+2 q}(u)\right] \\
& -\int_{-\infty}^{\infty}\left[2 a_{2}(u)+a_{4}(u)\right] \delta \rho_{\lambda q}(v) d v .
\end{aligned}
$$


Taking the Fourier transformation of Eq.(65), we obtain

$$
\delta \tilde{\rho}_{\lambda q}(\omega)=-\frac{1}{2 N} \frac{\tilde{a}_{1-2 q}(\omega)-\tilde{a}_{-1-2 q}(\omega)+\tilde{a}_{3-2 q}(\omega)+\tilde{a}_{3+2 q}(\omega)}{1+2 \tilde{a}_{2}(\omega)+\tilde{a}_{4}(\omega)}-\frac{1}{N} \frac{\cos \left(\omega \lambda_{0}\right) e^{-\frac{|\omega|}{2}}}{1+e^{-|\omega|}} .
$$

Then the deviation of energy $\delta e_{\lambda q}$ induced by $\delta \tilde{\rho}_{\lambda q}(\omega)$ is given by

$$
\begin{aligned}
\delta e_{\lambda q} & =-2 N \int_{-\infty}^{\infty}\left[\tilde{a}_{1}(\omega)+\tilde{a}_{3}(\omega)\right] \delta \tilde{\rho}_{\lambda q}(\omega) d \omega+\frac{4}{q^{2}-1}-\frac{4}{\lambda_{0}^{2}+1} \\
& =2 \int_{0}^{\infty} \frac{e^{-(2+q) \omega}}{1+e^{-\omega}} d \omega-2 \int_{0}^{\infty} \frac{e^{q \omega}}{1+e^{-\omega}} d \omega-\frac{2}{1+q}<0 .
\end{aligned}
$$

Due to $\delta e_{\lambda q}<0$, the ground state energy in this regime is $E_{g}=N e_{g}+e_{s}+\delta e_{\lambda q}$ and the total spin along the $z$-direction is $S_{z}=3 / 4$.

In the regime VI, there are $N-42$-string, one real Bethe root $\lambda_{0}$ and three boundary strings $q i, p i$ and $(p-1) i$. Combining the results (62) and (67), we obtain the ground state energy as $E_{g}=N e_{g}+e_{s}+\delta e_{p}+\delta e_{\lambda q}$.

After tedious calculation, we find that the boundary energy $e_{b}$ for all the regimes in Figure 1 can be expressed as

$$
e_{b}= \begin{cases}-\frac{2}{p}-\frac{2}{q}+2 \pi-4+E_{0}, & p>0, q>0 \text { or } q<-1, \\ -\frac{2}{p}-\frac{2}{q}+2 \pi \csc (q \pi)+2 \pi-4+E_{0}, & p>0,-1<q<0 .\end{cases}
$$

The boundary energies with different boundary parameters $p$ and $q$ calculated by the analytical expression (68) are shown in Figure 3 as the coloured solid lines. Now we check the correction of expression (68) by the numerical simulation with DMRG algorithm, and the results are shown in Figure 3 as the red points. Specifically, for each red point that is for the given boundary parameters $p$ and $q$, we first calculate the ground state energy $E_{g}(N)$ of the model (15) with the system size $N=10(n-1)+4$ and $n=1,2, \ldots, 20$ by using the DMRG method. Then we consider the physical quantity

$$
e_{b}(N)=E_{g}(N)-N e_{g}
$$

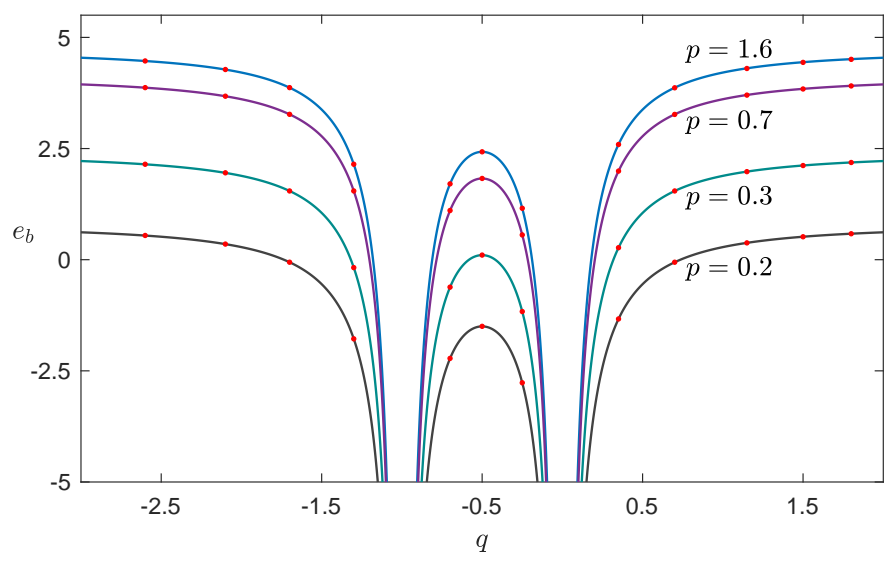

Figure 3: Boundary energies versus the boundary parameters $p$ and $q$. The coloured curves are those calculated from the analytical expression (68) and the red points are those obtained from the DMRG. The values of $q$ at the red points are $q=-2.6,-2.1,-1.7,-1.3,-0.7,-0.5,-0.25,0.35,0.7,1.15,1.5$ and 1.8 . 


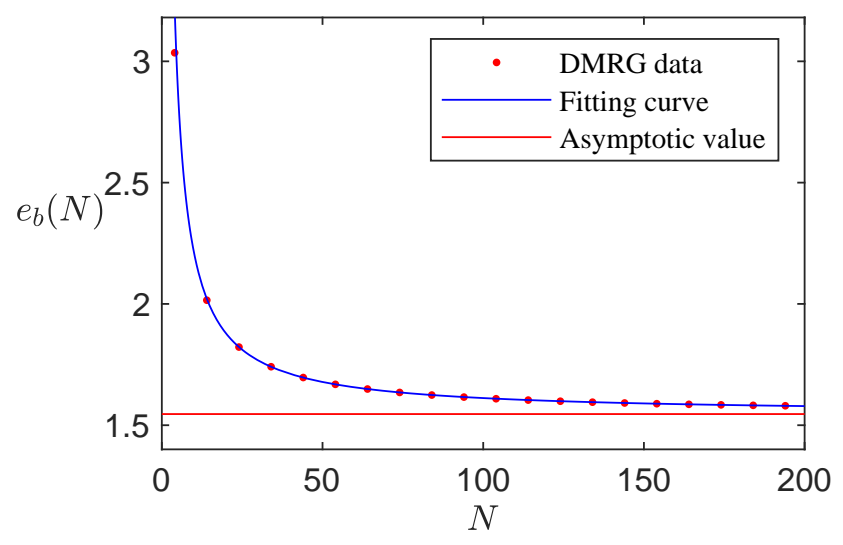

Figure 4: The values of $e_{b}(N)$ versus the system size $N$. The red points are the DMRG results with $N=4,14,24, \ldots, 194$. The data can be fitted as $e_{b}(N)=a N^{\beta}+c$, where $a=6.7308, \beta=-1.0046$ and $c=1.5460$. Due to the fact $\beta<0$, when the system size $N \rightarrow \infty$, the values of $e_{b}(N)$ tend to the asymptotic value $c$, which gives the boundary energy. Here the boundary parameters are chosen as $p=0.3$ and $q=0.7$.

where $e_{g}=-1$ is the ground state energy density of the system with periodic boundary conditions. Obviously, in the thermodynamic limit, the value of $e_{b}(N \rightarrow \infty)$ gives the boundary energy. In Figure 4, we show how to extrapolate the boundary energy, where the red points are the numerical values of $e_{b}(N)$, the blue solid line is the fitting curve, and the red solid line is the extrapolated boundary energy. From the fitting curve, we find that the $e_{b}(N)$ and $N$ satisfy the power law relation, i.e., $e_{b}(N)=a N^{\beta}+c$. Due to the fact that $\beta<0$, the values of $e_{b}(N)$ tend to the asymptotic value $c$ when the system size $N$ tends to infinity. Therefore, in the thermodynamic limit, the asymptotic value $c$ determines the boundary energy. Repeating this process, we obtain the boundary energies with other values of boundary parameters. As shown in Figure 3, the analytical and numerical results agree with each other very well.

\section{Conclusions}

In this paper, we have studied the thermodynamic limit and boundary energy of the isotropic spin-1 Heisenberg chain with generic integrable non-diagonal boundary reflections. It is shown that the contribution of the inhomogeneous term in the associated $T-Q$ relation (18) (due to the unparallel boundary fields) at the ground state can be neglected when the system size $N$ tend to infinity. Then we calculate the analytical expression of boundary energy (68) in the thermodynamic limit based on the string hypothesis of the reduced BAEs (29).

\section{Acknowledgments}

Financial support from the National Natural Science Foundation of China (Grant Nos. 12105221, 12175180, 12074410, 12047502, 11934015, 11975183 and 11947301), the Strategic Priority Research Program of the Chinese Academy of Sciences (Grant No. XDB33000000), the Major Basic Research Program of Natural Science of Shaanxi Province (Grant Nos. 2021JCW-19, 2017KCT-12 and 2017ZDJC-32), the Scientific Research Program Funded by Shaanxi Provincial Education Department (Grant No. 21JK0946), Beijing National Laboratory for Condensed Matter Physics (Grant No. 202162100001), and the Double First- 
Class University Construction Project of Northwest University is gratefully acknowledged. One of the authors, Zhihan Zheng would like to thank Dr. Yangyang Chen, Dr. Fakai Wen and Dr. Yi Qiao for their helpful discussions.

\section{References}

[1] X.-W. Guan, M. T. Batchelor and C. Lee, Fermi gases in one dimension: from Bethe ansatz to experiments, Rev. Mod. Phys. 85, 1633 (2013), doi:10.1103/RevModPhys.85.1633.

[2] M. Takahashi, Thermodynamics of one-dimensional solvable models, Cambridge University Press, Cambridge, UK, ISBN 9780521551434 (1999), doi:10.1017/CBO9780511524332.

[3] L. Dolan, C. R. Nappi and E. Witten, A relation between approaches to integrability in superconformal Yang-Mills theory, J. High Energy Phys. 10, 017 (2003), doi:10.1088/11266708/2003/10/017.

[4] J. Sirker, R. G. Pereira and I. Affleck, Diffusion and ballistic transport in one-dimensional quantum systems, Phys. Rev. Lett. 103, 216602 (2009), doi:10.1103/PhysRevLett.103.216602.

[5] J. de Gier and F. H. L. Essler, Bethe Ansatz solution of the asymmetric exclusion process with open boundaries, Phys. Rev. Lett. 95, 240601 (2005), doi:10.1103/PhysRevLett.95.240601.

[6] H. A. Bethe, Zur Theorie der Metalle. I. Eigenwerte und Eigenfunktionen der linearen Atomkette, Z. Phys. A 71, 205 (1931), doi:10.1007\%2FBF01341708.

[7] A. B. Zamolodchikov and V. A. Fateev, Model factorized S-matrix and an integrable Heisenberg chain with spin-1, Sov. J. Nucl. Phys. 32, 298 (1980).

[8] L. A. Takhtajan, The picture of low-lying excitations in the isotropic Heisenberg chain of arbitrary spins, Phys. Lett. A 87, 479 (1982), doi:10.1016/0375-9601(82)90764-2.

[9] H. M. Babujian, Exact solution of the isotropic Heisenberg chain with arbitrary spins: Thermodynamics of the model, Nucl. Phys. B 215, 317 (1983), doi:10.1016/05503213(83)90668-5.

[10] H. M. Babujian, Exact solution of the one-dimensional isotropic Heisenberg chain with arbitrary spins S, Phys. Lett. A 90, 479 (1982), doi:10.1016/0375-9601(82)90403-0.

[11] F. D. M. Haldane, Continuum dynamics of the 1-D Heisenberg antiferromagnet: Identification with the O(3) nonlinear sigma model, Phys. Lett. A 93, 464 (1983), doi:10.1016/0375-9601(83)90631-X.

[12] F. D. M. Haldane, Nonlinear field theory of large-spin Heisenberg antiferromagnets: Semiclassically quantized solitons of the one-dimensional easy-axis Néel state, Phys. Rev. Lett. 50, 1153 (1983), doi:10.1103/PhysRevLett.50.1153.

[13] I. Affleck, T. Kennedy, E. H. Lieb and H. Tasaki, Rigorous results on valencebond ground states in antiferromagnets, Phys. Rev. Lett. 59, 799 (1987), doi:10.1103/PhysRevLett.59.799. 
[14] I. Affleck, T. Kennedy, E. H. Lieb and H. Tasaki, Valence bond ground states in isotropic quantum antiferromagnets, Commun. Math. Phys. 115, 477 (1988), doi:10.1007/BF01218021.

[15] M. N. Barber and M. T. Batchelor, Spectrum of the biquadratic spin-1 antiferromagnetic chain, Phys. Rev. B 40, 4621 (1989), doi:10.1103/PhysRevB.40.4621.

[16] A. Klumper, The spectra of q-state vertex models and related antiferromagnetic quantum spin chains, J. Phys. A: Math. Gen. 23, 809 (1990), doi:10.1088/0305-4470/23/5/023.

[17] K.-J.-B. Lee and P. Schlottmann, Integrable spin-1 Heisenberg chain with impurity, Phys. Rev. B 37, 379 (1988), doi:10.1103/PhysRevB.37.379.

[18] F. C. Alcaraz, M. N. Barber, M. T. Batchelor, R. J. Baxter and G. R. W. Quispel, Surface exponents of the quantum XXZ, Ashkin-Teller and Potts models, J. Phys. A: Math. Gen. 20, 6397 (1987), doi:10.1088/0305-4470/20/18/038.

[19] E. K. Sklyanin, Boundary conditions for integrable quantum systems, J. Phys. A: Math. Gen. 21, 2375 (1988), doi:10.1088/0305-4470/21/10/015.

[20] P. P. Kulish and E. K. Sklyanin, Quantum spectral transform method recent developments, in Integrable Quantum Field Theories, Springer Berlin Heidelberg, ISBN 9783540111900 (1982), doi:10.1007/3-540-11190-5_8.

[21] P. P. Kulish, N. Yu. Reshetikhin and E. K. Sklyanin, Yang-Baxter equation and representation theory: 1, in Yang-Baxter equation in integrable systems, World Scientific, Singapore, ISBN 9789810201210 (1990), doi:10.1142/9789812798336_0027.

[22] P. P. Kulish and N. Yu. Reshetikhin, Quantum linear problem for the sine-Gordon equation and higher representations, J. Math. Sci. 23, 2435 (1983), doi:10.1007/BF01084171.

[23] A. N. Kirillov and N. Yu. Reshetikhin, Exact solution of the Heisenberg XXZ model of spin s, J. Math. Sci. 35, 2627 (1986), doi:10.1007/BF01083768.

[24] A. N. Kirillov and N. Yu Reshetikhin, Exact solution of the integrable XXZ Heisenberg model with arbitrary spin. I. The ground state and the excitation spectrum, J. Phys. A: Math. Gen. 20, 1565 (1987), doi:10.1088/0305-4470/20/6/038.

[25] M. Jimbo and T. Miwa, Algebraic analysis of solvable lattice models, American Mathematical Society, Providence, Rhode Island, ISBN 9780821803202 (1994), doi:10.1090/cbms/085.

[26] L. Mezincescu, R. I. Nepomechie and V. Rittenberg, Bethe ansatz solution of the FateevZamolodchikov quantum spin chain with boundary terms, Phys. Lett. A 147, 70 (1990), doi:10.1016/0375-9601(90)90016-H.

[27] E. C. Fireman, A. Lima-Santos and W. Utiel, Bethe ansatz solution for quantum spin1 chains with boundary terms, Nucl. Phys. B 626, 435 (2002), doi:10.1016/S05503213(02)00027-5.

[28] A. Doikou, Fused integrable lattice models with quantum impurities and open boundaries, Nucl. Phys. B 668, 447 (2003), doi:10.1016/j.nuclphysb.2003.07.001.

[29] O. A. Castro-Alvaredo and J. M. Maillet, Form factors of integrable Heisenberg (higher) spin chains, J. Phys. A: Math. Theor. 40, 7451 (2007), doi:10.1088/1751-8113/40/27/004. 
[30] C. S. Melo, G. A. P. Ribeiro and M. J. Martins, Bethe ansatz for the $X X X$ $S$ chain with non-diagonal open boundaries, Nucl. Phys. B 711, 565 (2005), doi:10.1016/j.nuclphysb.2004.12.008.

[31] L. Frappat, R. I. Nepomechie and E. Ragoucy, A complete Bethe ansatz solution for the open spin-s XXZ chain with general integrable boundary terms, J. Stat. Mech. P09009 (2007), doi:10.1088/1742-5468/2007/09/P09009.

[32] R. Murgan, Bethe ansatz of the open spin-s XXZ chain with nondiagonal boundary terms, J. High Energy Phys. 04, 076 (2009), doi:10.1088/1126-6708/2009/04/076.

[33] R. Baiyasi and R. Murgan, Generalized $T-Q$ relations and the open spin-s $X X Z$ chain with nondiagonal boundary terms, J. Stat. Mech. P10003 (2012), doi:10.1088/17425468/2012/10/P10003.

[34] R. I. Nepomechie, An inhomogeneous T-Q equation for the open XXX chain with general boundary terms: Completeness and arbitrary spin, J. Phys. A: Math. Theor. 46, 442002 (2013), doi:10.1088/1751-8113/46/44/442002.

[35] R. I. Nepomechie and C. Wang, Boundary energy of the open XXX chain with a nondiagonal boundary term, J. Phys. A: Math. Theor. 47, 032001 (2013), doi:10.1088/17518113/47/3/032001.

[36] E. K. Sklyanin, The quantum Toda chain, in Non-linear equations in classical and quantum field theory, Springer Berlin Heidelberg, ISBN 9783540152132 (1985), doi:10.1007/3540-15213-X_80.

[37] E. K. Sklyanin, Functional Bethe ansatz, in Integrable and superintegrable systems, World Scientific, Singapore, ISBN 9789810203160 (1990), doi:10.1142/9789812797179_0002.

[38] M. T. Batchelor, R. J. Baxter, M. J. O'Rourke and C. M. Yung, Exact solution and interfacial tension of the six-vertex model with anti-periodic boundary conditions, J. Phys. A: Math. Gen. 28, 2759 (1995), doi:10.1088/0305-4470/28/10/009.

[39] C. M. Yung and M. T. Batchelor, Exact solution for the spin-s XXZ quantum chain with non-diagonal twists, Nucl. Phys. B 446, 461 (1995), doi:10.1016/0550-3213(95)00168$\mathrm{R}$.

[40] H. Frahm, A. Seel and T. Wirth, Separation of variables in the open XXX chain, Nucl. Phys. B 802, 351 (2008), doi:10.1016/j.nuclphysb.2008.04.008.

[41] H. Frahm, J. H. Grelik, A. Seel and T. Wirth, Functional Bethe ansatz methods for the open XXX chain, J. Phys. A: Math. Theor. 44, 015001 (2010), doi:10.1088/1751$8113 / 44 / 1 / 015001$.

[42] G. Niccoli, Non-diagonal open spin-1/2 XXZ quantum chains by separation of variables: Complete spectrum and matrix elements of some quasi-local operators, J. Stat. Mech. P10025 (2012), doi:10.1088/1742-5468/2012/10/p10025.

[43] P. Baseilhac and S. Belliard, The half-infinite $X X Z$ chain in Onsager's approach, Nucl. Phys. B 873, 550 (2013), doi:10.1016/j.nuclphysb.2013.05.003.

[44] J. Cao, W.-L. Yang, K. Shi and Y. Wang, Off-diagonal Bethe ansatz and exact solution of a topological spin ring, Phys. Rev. Lett. 111, 137201 (2013), doi:10.1103/PhysRevLett.111.137201. 
[45] J. Cao, W.-L. Yang, K. Shi and Y. Wang, Off-diagonal Bethe ansatz solutions of the anisotropic spin-1/2 chains with arbitrary boundary fields, Nucl. Phys. B 877, 152 (2013), doi:10.1016/j.nuclphysb.2013.10.001.

[46] S. Belliard and N. Crampé, Heisenberg XXX model with general boundaries: Eigenvectors from algebraic Bethe ansatz, Symmetry Integr. Geom.: Methods Appl. (SIGMA) 9, 072 (2013), doi:10.3842/SIGMA.2013.072.

[47] N. Kitanine, J. M. Maillet and G. Niccoli, Open spin chains with generic integrable boundaries: Baxter equation and Bethe ansatz completeness from separation of variables, J. Stat. Mech. P05015 (2014), doi:10.1088/1742-5468/2014/05/P05015.

[48] G. Niccoli and V. Terras, Antiperiodic XXZ chains with arbitrary spins: Complete eigenstate construction by functional equations in separation of variables, Lett. Math. Phys. 105, 989 (2015), doi:10.1007/s11005-015-0759-9.

[49] S. Belliard, Modified algebraic Bethe ansatz for XXZ chain on the segment - I: Triangular cases, Nucl. Phys. B 892, 1 (2015), doi:10.1016/j.nuclphysb.2015.01.003.

[50] Y. Wang, W.-L. Yang, J. Cao and K. Shi, Off-diagonal Bethe ansatz for exactly solvable models, Springer Berlin Heidelberg, ISBN 9783662467558 (2015), doi:10.1007/978-3662-46756-5.

[51] R. J. Baxter, Generalized ferroelectric model on a square lattice, Stud. Appl. Math. 50, 51 (1971), doi:10.1002/sapm197150151.

[52] R. J. Baxter, Partition function of the eight-vertex lattice model, Ann. Phys. 70, 193 (1972), doi:10.1016/0003-4916(72)90335-1.

[53] N. Yu. Reshetikhin, The functional equation method in the theory of exactly soluble quantum systems, Sov. Phys. JETP 57, 691 (1983).

[54] Y.-Y. Li, J. Cao, W.-L. Yang, K. Shi and Y. Wang, Thermodynamic limit and surface energy of the XXZ spin chain with arbitrary boundary fields, Nucl. Phys. B 884, 17 (2014), doi:10.1016/j.nuclphysb.2014.04.010.

[55] F. Wen, T. Yang, Z. Yang, J. Cao, K. Hao and W.-L. Yang, Thermodynamic limit and boundary energy of the su(3) spin chain with non-diagonal boundary fields, Nucl. Phys. B 915, 119 (2017), doi:10.1016/j.nuclphysb.2016.12.003.

[56] S. R. White, Density matrix formulation for quantum renormalization groups, Phys. Rev. Lett. 69, 2863 (1992), doi:10.1103/PhysRevLett.69.2863.

[57] S. R. White, Density-matrix algorithms for quantum renormalization groups, Phys. Rev. B 48, 10345 (1993), doi:10.1103/PhysRevB.48.10345.

[58] U. Schollwöck, The density-matrix renormalization group, Rev. Mod. Phys. 77, 259 (2005), doi:10.1103/RevModPhys.77.259.

[59] C. N. Yang, Some exact results for the many-body problem in one dimension with repulsive delta-function interaction, Phys. Rev. Lett. 19, 1312 (1967), doi:10.1103/PhysRevLett.19.1312.

[60] R. J. Baxter, Exactly solved models in statistical mechanics, in Series on advances in statistical mechanics, World Scientific, Singapore, ISBN 9789971978112 (1985), doi:10.1142/9789814415255_0002. 
[61] L. Mezincescu, R. I. Nepomechie and V. Rittenberg, Bethe ansatz solution of the FateevZamolodchikov quantum spin chain with boundary terms, Phys. Lett. A 147, 70 (1990), doi:10.1016/0375-9601(90)90016-H.

[62] T. Inami, S. Odake and Y.-Z. Zhang, Reflection K-matrices of the 19-vertex model and $X X Z$ spin-1 chain with general boundary terms, Nucl. Phys. B 470, 419 (1996), doi:10.1016/0550-3213(96)00133-2.

[63] A. Kapustin and S. Skorik, Surface excitations and surface energy of the antiferromagnetic XXZ chain by the Bethe ansatz approach, J. Phys. A: Math. Gen. 29, 1629 (1996), doi:10.1088/0305-4470/29/8/011.

[64] H. J. de Vega and F. Woynarovich, Method for calculating finite size corrections in Bethe ansatz systems: Heisenberg chain and six-vertex model, Nucl. Phys. B 251, 439 (1985), doi:10.1016/0550-3213(85)90271-8.

[65] A. Klumper, M. T. Batchelor and P. A. Pearce, Central charges of the 6- and 19-vertex models with twisted boundary conditions, J. Phys. A: Math. Gen. 24, 3111 (1991), doi:10.1088/0305-4470/24/13/025.

[66] J. Suzuki, Spinons in magnetic chains of arbitrary spins at finite temperatures, J. Phys. A: Math. Gen. 32, 2341 (1999), doi:10.1088/0305-4470/32/12/008.

[67] M. Gaudin, Boundary energy of a Bose gas in one dimension, Phys. Rev. A 4, 386 (1971), doi:10.1103/PhysRevA.4.386.

[68] C. J. Hamer, G. R. W. Quispel and M. T. Batchelor, Conformal anomaly and surface energy for Potts and Ashkin-Teller quantum chains, J. Phys. A: Math. Gen. 20, 5677 (1987), doi:10.1088/0305-4470/20/16/040.

[69] M. T. Batchelor and C. J. Hamer, Surface energy of integrable quantum spin chains, J. Phys. A: Math. Gen. 23, 761 (1990), doi:10.1088/0305-4470/23/5/019.

[70] P. Fendley and H. Saleur, Deriving boundary S matrices, Nucl. Phys. B 428, 681 (1994), doi:10.1016/0550-3213(94)90369-7. 